\title{
Fabrication and Characteristics of Energetic Igniters Realized Through Sputtered Tantalum Nitride(TaN) Film Bridge
}

\author{
Yan Yichao \\ State Key Laboratory of Electronic Thin Films and \\ Integrated Devices \\ University of Electronic Science and Technology of \\ China, \\ Chengdu, China \\ e-mail: yanyichao@uestc.edu.cn
}

Xiong Jie

State Key Laboratory of Electronic Thin Films and Integrated Devices

University of Electronic Science and Technology of China,

Chengdu, China

e-mail:jiexiong@uestc.edu.cn

\author{
Jiang Hongchuan \\ State Key Laboratory of Electronic Thin Films and \\ Integrated Devices \\ University of Electronic Science and Technology of \\ China, \\ Chengdu, China \\ e-mail: hcjiang@uestc.edu.cn \\ Zhang Wanli \\ State Key Laboratory of Electronic Thin Films and \\ Integrated Devices \\ University of Electronic Science and Technology of \\ China, \\ Chengdu, China \\ e-mail:wlzhang@uestc.edu.cn
}

\begin{abstract}
This paper introduces energetic igniters fabricated using TaN film bridge by magnetron sputtering. It is inferred from the image of wet-etched TaN film bridge that film edge is clearly visible and relatively smooth with lateral erosion size of $5 \mu \mathrm{m}$. The X-ray diffraction (XRD) and Temperature Coefficient of Resistance (TCR) results show that the crystalline of TaN films has great influence on the TCR values by changing the $\mathrm{N}_{2}$ partial pressure. The TaN films in this paper exhibit a near zero TCR value of approximately $10 \mathrm{ppm} /{ }^{\circ} \mathrm{C}$. The electrical explosion characteristics employing a capacitor discharge firing set at the optimized charging voltage of $45 \mathrm{~V}$ reveal that an excellent explosion phenomenon of $\mathrm{TaN}$ film bridge is observed with small ignition delay time, high explosion temperature, much more bright flash of light and much large quantities of the ejected product particles. The peak explosive temperature of TaN film bridge at various dimensions is ranging from $3500 \mathrm{~K}$ to $5700 \mathrm{~K}$, which is almost as same as that of the semiconductor bridge.
\end{abstract}

Keywords-TaN; Film Bridge; Plasma; Temperature Coefficient of Resistance; Electrical Explosion Characteristic

\section{INTRODUCTION}

With the increasing demand for small ignition devices, investigation of heavily semiconductor or metallic film bridge, especially for doped polycrystalline silicon, titanium, platinum and chromium bridge, has attracted much attention in recent years [1-7]. Film ignition bridge devices, which are over 30 times smaller in volume, can function in a few tens of microseconds and operate at onetenth the input energy compared with the hot-wire devices while improving no-fire conditions and electrostatic safety. In the discharge mechanism of ignition film bridge, thermal plasma is generated to ignite explosive powder by passing current through the bridge which in turn change the physical features of the bridge.

Due to its excellent physical properties including quite high values sheet resistance [8], tantalum nitride(TaN) can be a promising candidate for high precision thin film resistors The adjusted temperature coefficient of resistance (TCR) characteristics of the film by changing the sputtering parameters make it ideally suited for application across a large temperature range compared with metallic film bridge devices.

In this paper, a primary object is to design and fabricate an energetic initiator realized using TaN film bridge based on anisotropic wet etching. In order to elucidate the relation between the crystalline and TCR characteristics of $\mathrm{TaN}$ film, the microstructure characterization and TCR analysis are described in detail. The electrical explosion properties and ignition flame temperature of TaN film bridge are also investigated.

\section{EXPERIMENTAL}

A schematic diagram of the experimental TaN film ignition bridge is shown in Fig. 1. It consists of a wetetched "H" shaped thin TaN polycrystalline film deposited by magnetron sputtering, and two metal lands of copper attached to the membrane. The dimensions of bridge are $80 \mu \mathrm{m}$ long (l) by $40 \mu \mathrm{m}$ wide (w) by $2 \mu \mathrm{m}$ thick $(\mathrm{t})$, which will influence the energy loss into the substrate. Two metal lands are designed to maximize the 
metal/TaN contact area to insure much less contact resistances.

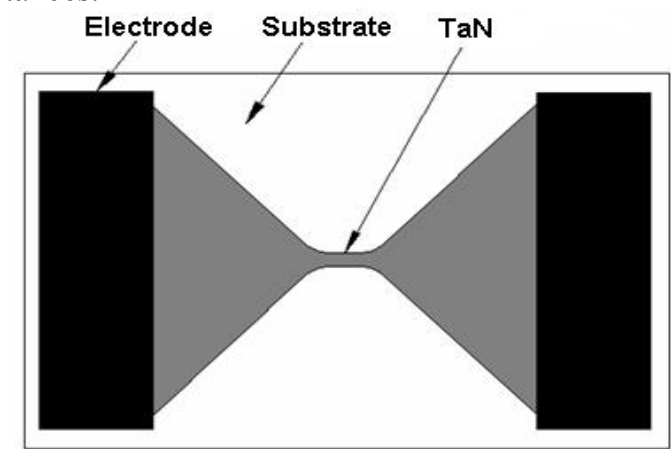

Figure 1. Schematic view of an initiator of TaN film bridge

The TaN films are deposited onto single side polished alumina substrate $(10 \mathrm{~mm} \times 5 \mathrm{~mm} \times 0.5 \mathrm{~mm})$ by D.C. magnetron sputtering. Before deposition, the substrates are cleaned with acetone, alcohol and de-ionized water in an ultrasonic bath for $10 \mathrm{~min}$, respectively, which subsequently are dried by nitrogen gas and placed in the oven of $200{ }^{\circ} \mathrm{C}$ for $2 \mathrm{~h}$. A tantalum target (99.995\% purity) of diameter $60 \mathrm{~mm}$ is attached to the sputter source. The rotary sample holder is located at the center of the chamber, with the distance being $80 \mathrm{~mm}$ between target and substrate, which is considered to provide the best deposition rate and uniformity of thin films. When the base pressure in the vacuum chamber is pumped down to $5 \times 10^{-4} \mathrm{~Pa}$, before the deposition of the TaN, argon $(99.999 \%)$ gas is firstly introduced into the chamber using mass flow controllers as sputtering gas, a thin interlayer of tantalum is deposited for $20 \mathrm{~min}$, and subsequently nitrogen $(99.999 \%)$ gas is introduced into the chamber as reactive gas. To determine the effect of nitrogen partial pressure, the percentage of $\mathrm{N}_{2} /\left(\mathrm{N}_{2}+\mathrm{Ar}\right)$ gas ratio is varied from $0.33 \%$ to $2 \%$. Sputtering temperature, pressure, power and time are fixed to $200{ }^{\circ} \mathrm{C}, 0.33 \mathrm{~Pa}, 120 \mathrm{~W}, 30$ min, respectively. After the deposition, reversal photoresist (PRI-9000A) is spin-coated onto TaN films and patterned using photolithography through a designed mask. Once the photoresist is exposed, deposited TaN film is rinsed in developer and then placed in the oven of $100{ }^{\circ} \mathrm{C}$ for $1 \mathrm{~h}$. Subsequently, the exposed TaN film is directly wet-etched in the corrosive liquid. As can be seen in Fig. 2, film edge is clearly visible and relatively smooth, there is no obvious defects and burrs. Fig. 3 is a crosssectional scanning electron microscope (SEM) image of TaN film bridge with the lateral erosion size of $5 \mu \mathrm{m}$. The crystallographic structure of the TaN films is determined by Bede D1 X-ray diffraction (XRD) using $\mathrm{Cu} \mathrm{Ka}$ radiation. Film resistance is calculated from the sheet resistance measured by a SDY-4 four-point probe and the film thickness is measured by a Dektak150 profilometer. The temperature coefficient of resistance (TCR) values of TaN films are achieved in a fixed set of apparatus in our lab.

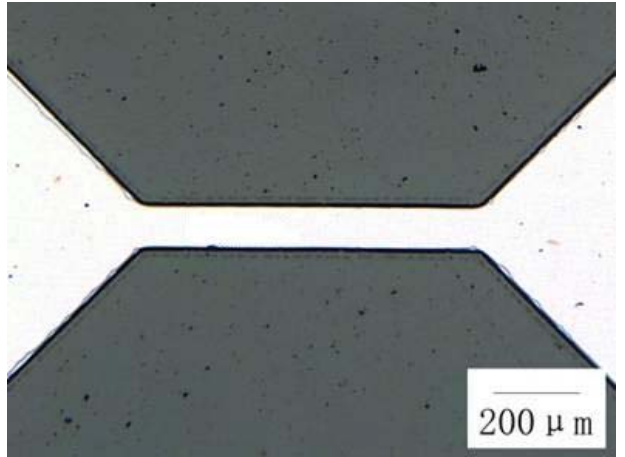

(a)Top-view

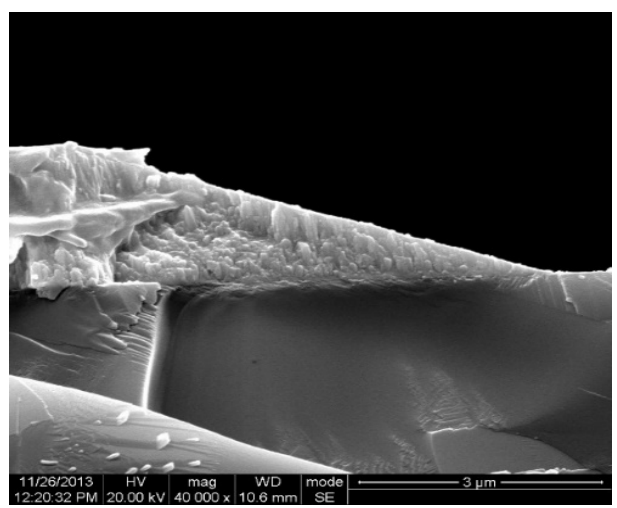

(b) cross-sectional

Figure 2. SEM image of TaN film bridge

The principle of open-air electrical explosion testing of the initiator is introduced in detail in a few literature[911], according to the literature, in this paper, the parameter including the ignition voltage, ignition current and ignition delay are achieved by using capacitor voltage discharging firing set. The explosion temperature of the initiator is determined based on the "double-line atomic emission spectroscopy of a copper element" with the fundamental principle [9-10]. A high speed camera (HS4540MX12) with 20000 frames per second resolution is used to observe the explosion process directly.

III. RESULTS AND DISCUSSION

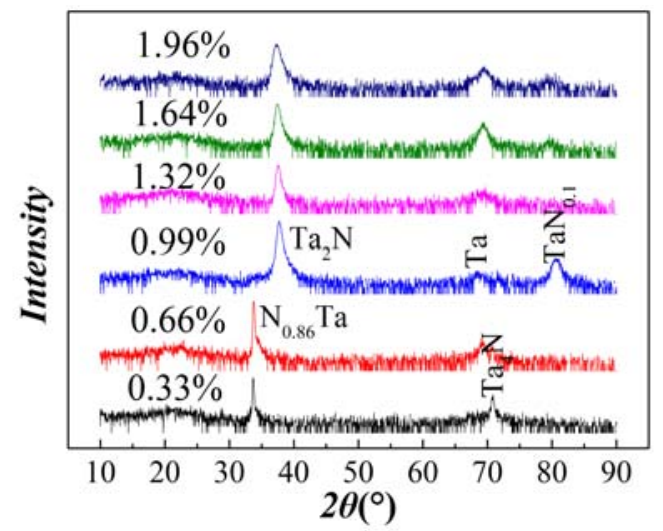

Figure 3. XRD spectra of TaN films deposited on the alumina substrate with various $\mathrm{N}_{2} /\left(\mathrm{N}_{2}+\mathrm{Ar}\right)$ gas ratios

As shown in Fig. 3, when other deposition parameters including deposition pressure, temperature and power remain constant, the main phases of the films evolve from 
Ta to $\mathrm{Ta}_{\mathrm{x}} \mathrm{N}$ by changing the nitrogen gas ratio. At low $\mathrm{N}_{2} /\left(\mathrm{N}_{2}+\mathrm{Ar}\right)$ gas ratio, only $\mathrm{TaN}_{0.86}$ and $\mathrm{Ta}$ phases are observed. With the increase of the nitrogen gas ratio form $0.33 \%$ to $1.96 \%$, TaN film consists of different phases. At the $\mathrm{N}_{2} /\left(\mathrm{N}_{2}+\mathrm{Ar}\right)$ gas ratio of $1.96 \%, \mathrm{Ta}_{2} \mathrm{~N}$ phase is only present. Although N-rich phases are absent in the XRD spectra, the increased resistivity as a function of the nitrogen gas ratio in the $\mathrm{TaN}$ film demonstrates the existence of $\mathrm{N}$-rich phase.

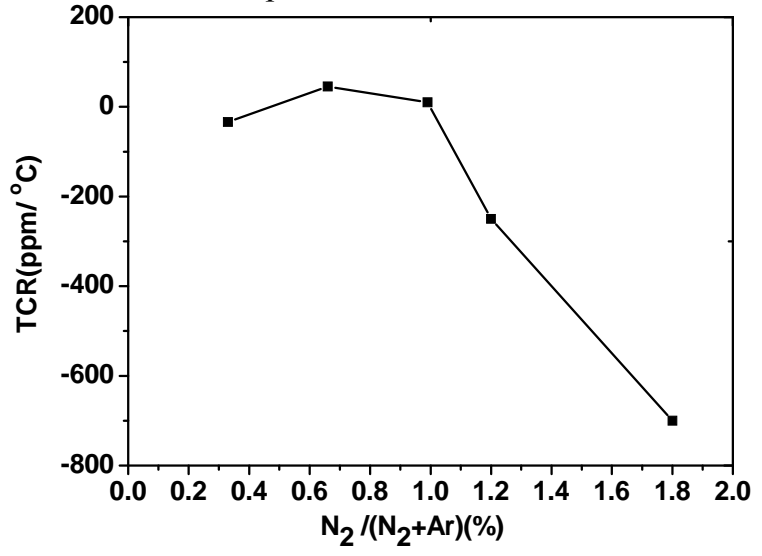

Figure 4. The temperature coefficient of resistance of TaN films as a function of nitrogen gas ratio

The temperature coefficient of resistance of TaN films in Fig. 4 decreases with an increase of nitrogen gas ratio. The difference in TCR as a function of nitrogen gas ratio is mainly affected by film phases. The near zero TCR corresponds to the $\mathrm{TaN}_{0.86}$ existence when the nitrogen gas ratio is below $1 \%$, however, the appearance of $\mathrm{Ta}_{2} \mathrm{~N}$ phase leads to much larger value of negative TCR with the nitrogen ratio shifting from $1 \%$ to $1.96 \%$. Due to the presence of Ta phase in the XRD spectra, a further reduced TCR value of TaN film could be achieved, because the positive value TCR of the Ta phase can counteract the $\mathrm{Ta}_{2} \mathrm{~N}$ phase with negative TCR. It may be ignited with relatively low electrical input voltage while the resistance remains between $1 \Omega$ and $4 \Omega$, furthermore, much smaller and even near zero TCR for TaN film with low resistivity is favorable for fabrication process. Nitrogen gas ratio of $0.99 \%$ is chosen in this paper at which the TaN films exhibit a near zero TCR value of approximately $10 \mathrm{ppm} /{ }^{\circ} \mathrm{C}$.

For the evaluation of electrical explosion behaviors, a capacitor discharge firing circuit with various charged voltages $(47 \mu \mathrm{F}, 45 \mathrm{~V}, 40 \mathrm{~V}, 25 \mathrm{~V}$ or $15 \mathrm{~V})$ is adopted to apply the currents across the film bridge with an electrical trigger pulse and all parameters can be measured simultaneously. There is a corresponding optimized discharging voltage for a specific structure of film bridge, the bridge film can't obtain sufficient energy to explode thoroughly for a relatively lower capacitor charged voltage. Therefore, the comparison and analysis of electrical explosion behaviors with different capacitor charged voltage is significant for our investigation.

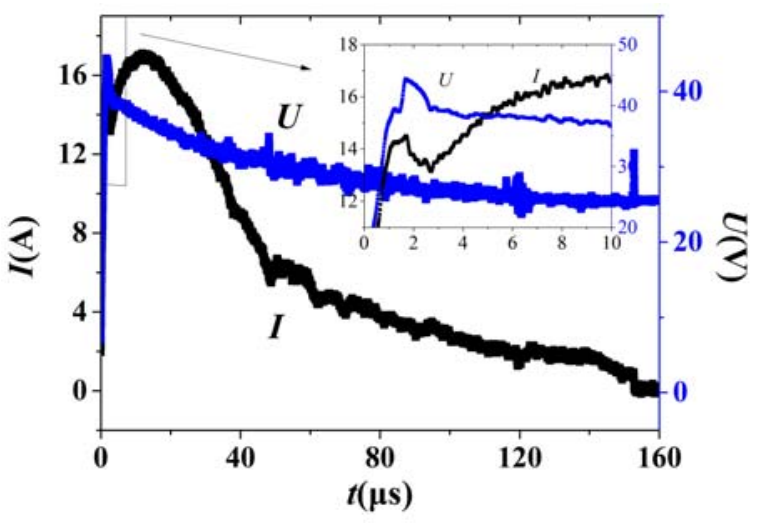

(a) $45 \mathrm{~V}$

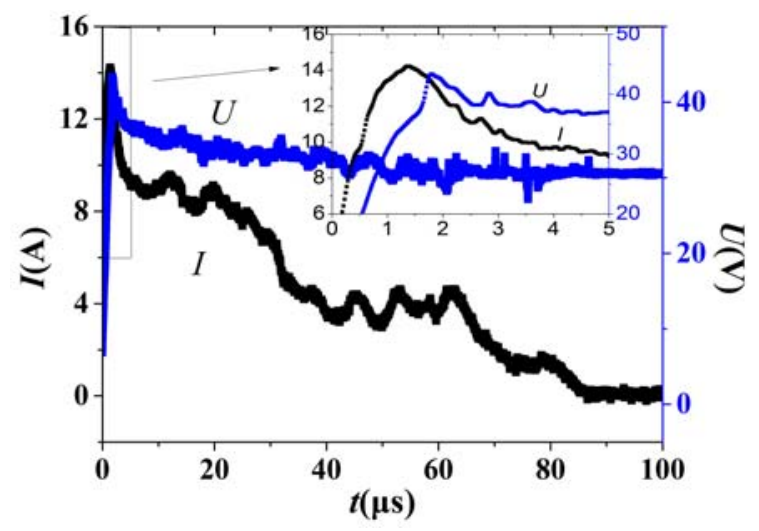

(b) $40 \mathrm{~V}$

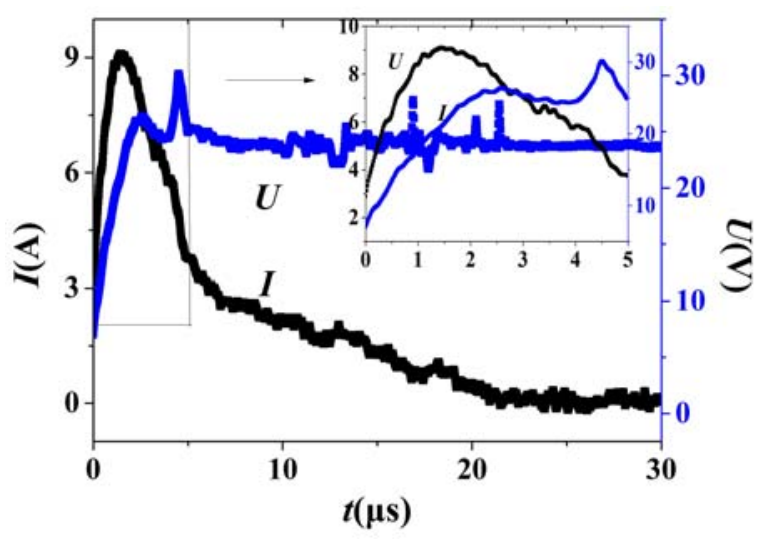

(c) $25 \mathrm{~V}$

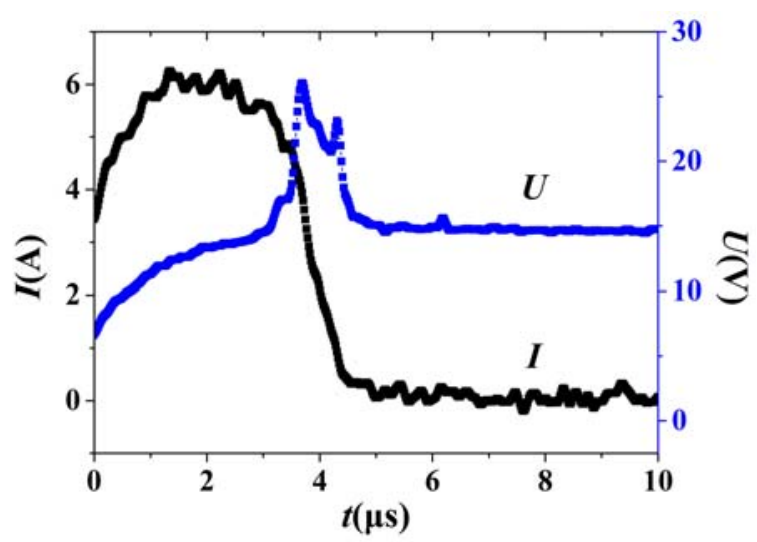

(d) $15 \mathrm{~V}$

Figure 5. Voltage-current histories for TaN film with various charged voltage 
Fig. 5 shows the variation of voltage and current as a function of time with the capacitor voltage charged to $45 \mathrm{~V}$, $40 \mathrm{~V}, 25 \mathrm{~V}$ and $15 \mathrm{~V}$, respectively. By comparison of the voltage-current curves of $\mathrm{TaN}$ film, $45 \mathrm{~V}$ is considered as the optimized charging voltage at which the voltage and current curves almost reach the peak simultaneously. The time of reaching the voltage peak value at $45 \mathrm{~V}$ is almost within $2 \mu \mathrm{s}$, which means the rapid melting, vaporization and ionization process of the TaN material. At the same time, the ignition duration time for $45 \mathrm{~V}$ is almost two times as that of $\mathrm{TaN}$ film for $40 \mathrm{~V}$, which gives rise to more ionized bridge materials.

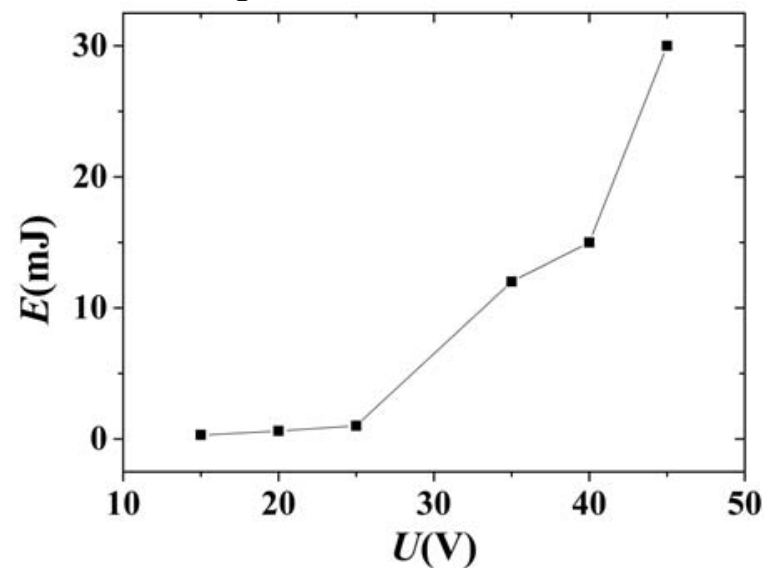

Figure 6. The input energy as a function of input voltage of TaN film bridge

The input energies which are applied on the film bridge are calculated by integrating the drop of voltage on the film bridge and the current flowing through it. As shown in Fig. 6, the input energy increases almost linearly with an increase of input voltage, which indicates that more energy will be imposed on the film bridge. It can be confirmed in Fig. 8, when the current pulse flows through the film bridge, a large fraction of current is gathered in the center regions of bridge due to the relatively low resistance, which begin to melt and vaporize to produce a weakly ionized hot plasma firstly because of the biggest energy densities and the fastest rising rate of the corresponding explosive temperature. As the input energy increases, the maximum temperature regions will rapidly spreading outward along the bridge, more and more regions of $\mathrm{TaN}$ film bridge are heated and burst into hot plasmas, which will readily ignite the explosives in a much shorter time.

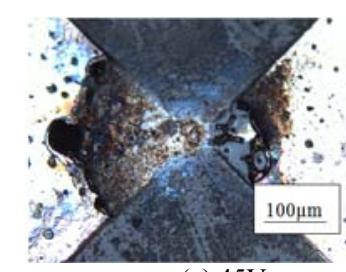

(a) $45 \mathrm{~V}$

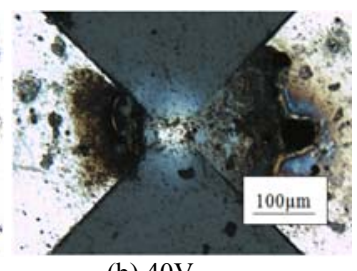

(b) $40 \mathrm{~V}$

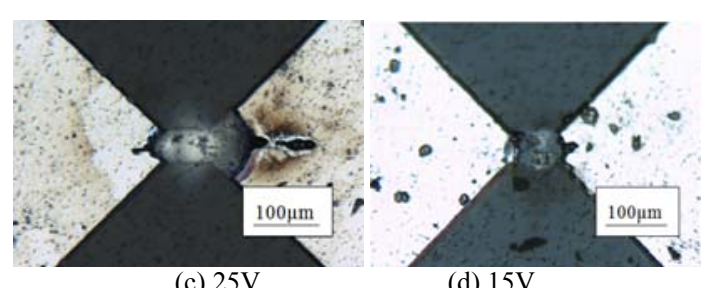

(c) $25 \mathrm{~V}$

(d) $15 \mathrm{~V}$

Figure 7. Top-view image of TaN film after electrical explosive test
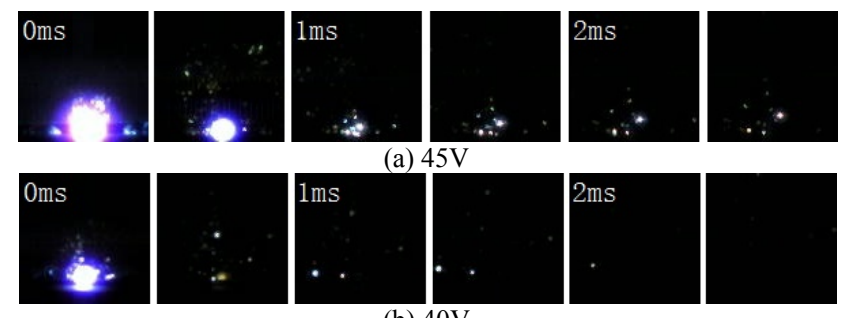

(b) $40 \mathrm{~V}$

Figure 8 . High speed camera observation of electrical explosion process for TaN film

As shown in Fig. 7 and Fig. 8, it shows the electrical explosive process from the beginning of the plasma discharge and the plasma shape seems to be uneven in the horizontal and vertical directions. It indicates that as the input voltage increases, the generated plasma along the horizontal and vertical direction increases and the plasma discharge duration becomes longer. It is noted that a fiercer explosion process is observed accompanied with much more bright flash of light, much larger quantities and longer distance of the ejected product particles by comparison with low charged voltage. It can be clearly seen that with the increase of the time, the plasma emission intensity correspondingly decreases for a specific input voltage, which is attributed to the plasma energy transfer by thermal diffusion or thermal pulse shock from hot plasma core to the relatively cooled ambient air encircling the plasma.

\section{CONCLUSIONS}

The purpose of this work is to gain a better understanding of the electrical explosion characteristics of TaN film ignition devices. Excellent temperature stability by adjusting $\mathrm{N}_{2} / \mathrm{Ar}$ flow ratio and high melting temperature makes it ideally suited for application across a large temperature range in the plasma discharge devices. From the electrical explosion characteristics of TaN film at various input voltage, it is observed that the input voltage and current of TaN film reach the peak value at 45 $\mathrm{V}$ almost simultaneously. It is definitely clear that as the input voltage increases, a much larger quantities of TaN film bridge materials are vaporized and ionized, testifying in the high speed camera that the size of the generated hot plasma increases corresponding with the increase of the input voltage. Much higher input voltage means a smaller ignition response time, a greater output energies and a high peak explosive temperature.

In a word, the advantage of low input ignition energy, the adjustable temperature coefficient of resistance at any time, smaller response time and high temperature ejected product particles makes TaN film an ideal choice as a substitute of semiconductor bridge materials. 


\section{REFERENCES}

[1] D. A. Benson, M. E. Larsen, A. M. Renlund. "Semiconductor bridge: A plasma generator for the ignition of explosives," Journal of applied physics, vol 62, pp. 1622-1632, 1987

[2] J. Kim, C. Park, M. Park. "Characteristics of semiconductor bridge (SCB) plasma generated in a micro-electro-mechanical system (MEMS)," Physics Letters A, vol 305, pp. 413-418, 2002

[3] M. Park, H. Choo, S. Yoon. "Comparison of plasma generation behaviors between a single crystal semiconductor bridge (singleSCB) and a polysilicon semiconductor bridge (poly-scb),". Sensors and Actuators A, vol 115, pp. 104-108, 2004

[4] K. Lee, M. Park, S. Choi,. "Characteristics of plasma generated by polysilicon semiconductor bridge (SCB)," Sensors and Actuators A vol 1196, pp. 252-257, 2002

[5] G. F. Zhang, Z. You, S. Hu, "MEMS-based propulsion arrays with solid propellant," Journal of Tsinghua University (Science and Technology), vol 44, pp. 1489-1492, 2002
[6] K. L. Zhang, S. K. Chou, S. S. Ang. "A MEMS-based solid propellant microthruster with $\mathrm{Au} / \mathrm{Ti}$ igniter," Sensors and Actuators A: Physical, vol 122, pp. 113-123, 2005

[7] X. Z. Wu, P. T. Dong, Z. Z. Li. "Design, fabrication and characterization of a solidpropellant micro-Thruster," Nano/Micro engineered and molecular systems, vol 13, pp. 476-479, 2009

[8] T. Riekkinen, J. Molarius, T. Laurila. "Reactive sputter deposition and properties of TaxN thin films," Microelectronic Engineering, 2002, vol 64, pp. 289-297, 2002

[9] P. Zhu, R. Shen, Y. Ye. "Energetic igniters realized by integrating $\mathrm{Al} / \mathrm{CuO}$ reactive multilayer films with $\mathrm{Cr}$ films," Journal of Applied Physics, vol 110, pp. 074513, 2011

[10] X. Zhou, R. Shen, Y. Ye. "Influence of $\mathrm{Al} / \mathrm{CuO}$ reactive multilayer films additives on exploding foil initiator," Journal of Applied Physics, vol 110: pp. 094505, 2011,

[11] P. Zhu, R. Shen, Y. Ye. "Characterization of $\mathrm{Al} / \mathrm{CuO}$ nanoenergetic multilayer films integrated with semiconductor bridge for initiator applications," Journal of Applied Physics, vol 113 :pp. 